\title{
ON THE CONVOLUTION OF $L_{2}$ FUNCTIONS
}

\author{
By SABUROU SAITOH
}

\section{Introduction.}

For the convolution $F^{*} G$ of $F \in L_{p}(-\infty, \infty)(p \geqq 1)$ and $G \in L_{1}(-\infty, \infty)$, we know the fundamental inequality

$$
\left\|F^{*} G\right\|_{p} \leqq\|F\|_{p}\|G\|_{1} .
$$

See, for example, $\left[8\right.$, p. 3]. Note that for $F, G \in L_{2}(-\infty, \infty)$, in general, $F * G \notin$ $L_{2}(-\infty, \infty)$. In this paper, we will give an identification of a Hilbert space spanned by the convolutions $F^{*} G$ and establish fundamental inequalities in the convolution. Note that when the space is $L_{2}(0, \infty)$, the results are very simple and quite different from the present case $L_{2}(-\infty, \infty)$. See [7].

\section{The case of functions with compact supports.}

We first consider the case of the convolution $F^{*} G$ of $F \in L_{2}(a, b)$ and $G \in$ $L_{2}(c, d)$. Without loss of generality we assume that $a+d \leqq b+c$. Of course, in the convolution we regard $F$ and $G$ as zero in the outsides of the intervals $[a, b]$ and $[c, d]$, respectively. We consider the integral transform, for $F \in L_{2}(a, b)$ and $z=x+i y \in \boldsymbol{C}$

$$
f(z)=\frac{1}{2 \pi} \int_{a}^{b} F(t) e^{-\imath z t} d t .
$$

As we see from the general theory $[5,6]$ of integral transforms, the images $f(z)$ form the Hilbert space $H_{(a, b)}$ admitting the reproducing kernel on $\boldsymbol{C}$

$$
K_{(a, b)}(z, \bar{u})=\frac{1}{2 \pi} \int_{a}^{b} e^{-\imath z t} e^{i u t} d t .
$$

Since the family $\left\{e^{-\imath z t} ; z \in \boldsymbol{C}\right\}$ is complete in $L_{2}(a, b)$, we further have the isometrical identity

$$
\|f\|_{H(a, b)}^{2}=\frac{1}{2 \pi} \int_{a}^{b}|F(t)|^{2} d t .
$$

Hence, by using the Fourier transform for (2.1) in the framework of the $L_{2}$ space, we have

Received March 16, 1985 


$$
\|f\|_{H(a, b)}^{2}=\frac{1}{2 \pi} \int_{a}^{a}\left|\operatorname{lid} \cdot \lim _{N \rightarrow \infty} \int_{-N}^{N} f(x) e^{\imath x t} d x\right|^{2} d t .
$$

We consider similarly the integral transform, for $G \in L_{2}(c, d)$

$$
g(z)=\frac{1}{2 \pi} \int_{c}^{d} G(t) e^{-\imath z t} d t
$$

and the Hilbert space $H_{(c, d)}$ admitting the reproducing kernel

$$
K_{(c, d)}(z, \bar{u})=\frac{1}{2 \pi} \int_{c}^{d} e^{-\imath z t} e^{i \bar{u} t} d t .
$$

Then, we have

$$
f(z) g(z)=\frac{1}{4 \pi^{2}} \int_{a+c}^{b+d}\left(F^{*} G\right)(t) e^{-\imath z t} d t
$$

where

$$
(F * G)(t)=\left\{\begin{array}{lll}
\int_{a}^{t-c} F\left(t_{1}\right) G\left(t-t_{1}\right) d t_{1} & \text { for } & a+c \leqq t \leqq a+d \\
\int_{t-d}^{t-c} F\left(t_{1}\right) G\left(t-t_{1}\right) d t_{1} & \text { for } & a+d \leqq t \leqq b+c \\
\int_{t-d}^{b} F\left(t_{1}\right) G\left(t-t_{1}\right) d t_{1} & \text { for } & b+c \leqq t \leqq b+d .
\end{array}\right.
$$

The product $f(z) g(z)$ belongs to the Hilbert space $\left[H_{(a, b)} \otimes H_{(c, d)}\right]_{R}$ which is the restriction of the tensor product $H_{(a, b)} \otimes H_{(c, d)}$ to the diagonal set $\boldsymbol{C}$ of $\boldsymbol{C} \times \boldsymbol{C}$. Here the norm is given by

$$
\left.\|f g\|_{[H(a, b)}^{2} \otimes H(c, d)\right]_{R}=\min \sum_{\jmath=1}^{\infty} \sum_{k=1}^{\infty}\left(f_{\jmath}, f_{k}\right)_{H(a, b)}\left(g_{\jmath}, g_{k}\right)_{I I(c, d)} .
$$

The minimum is taken over all functions $\sum_{j=1}^{\infty} f_{j}\left(z_{1}\right) g_{j}\left(z_{2}\right)$ on $\boldsymbol{C} \times \boldsymbol{C}$ satisfying

$$
f(z) g(z)=\sum_{j=1}^{\infty} f_{j}(z) g_{j}(z) \text { on } \boldsymbol{C}
$$

for $f_{j} \in H_{(a, b)}$ and $g_{j} \in H_{(c, d)}$. Moreover, the Hilbert space $\left[H_{(a, b)} \otimes H_{(c, d)}\right]_{R}$ admits the reproducing kernel $K_{(a, b)}(z, \bar{u}) K_{(c, d)}(z, \bar{u})$ and is characterized by this property ([1, pp. 357-362 and p. 344]).

In order to realize the norm in $\left[H_{(a, b)} \otimes H_{(c, d)}\right]_{R}$, we compute the kernel $K_{(a, b)}(z, \bar{u}) K_{(c, d)}(z, \bar{u})$ in a reduced form; that is,

$$
\begin{aligned}
& K_{(a, b)}(z, \bar{u}) K_{(c, d)}(z, \bar{u}) \\
& \quad=\frac{1}{4 \pi^{2}} \int_{a}^{b} \int_{c}^{d} e^{-\imath z t_{1}} e^{i u t_{1}} e^{-\imath z t_{2}} e^{\imath u t_{2}} d t_{1} d t_{2}
\end{aligned}
$$




$$
\begin{aligned}
= & \frac{1}{4 \pi^{2}} \int_{a+c}^{a+d}\{t-(a+c)\} e^{-\imath z t} e^{i u t} d t \\
& +\frac{1}{4 \pi^{2}} \int_{a+d}^{b+c}(d-c) e^{-\imath z t} e^{i u t} d t \\
& +\frac{1}{4 \pi^{2}} \int_{b+c}^{b+d}\{(b+d)-t\} e^{-\imath z t} e^{i u t} d t .
\end{aligned}
$$

We denote, in general, the characteristic function of $[a, b]$ by $\chi(t ;[a, b])$ such that

We set

$$
\chi(t ;[a, b])= \begin{cases}1 & \text { for } t \in[a, b] \\ 0 & \text { for } t<a, \text { or } b<t .\end{cases}
$$

$$
\begin{aligned}
V(t)= & \{t-(a+c)\} \chi(t ;[a+c, a+d])+(d-c) \chi(t ;[a+d, b+c]) \\
& +(b+d-t) \chi(t ;[b+c, b+d]) .
\end{aligned}
$$

Then, any member $\phi(z)$ of $\left[H_{(a, b)} \otimes H_{(c, d)}\right]_{R}$ is expressible in the form

$$
\phi(z)=\frac{1}{4 \pi^{2}} \int_{-\infty}^{\infty} \Phi(t) e^{-\imath z t} V(t) d t,
$$

for a uniquely determined function $\Phi$ satisfying

$$
\int_{-\infty}^{\infty}|\Phi(t)|^{2} V(t) d t<\infty
$$

Moreover, the norm is given by, as in (2.4)

$$
\begin{aligned}
\|\phi\|_{[H(a, b) \otimes H(c, d)]_{R}}^{2} & =\frac{1}{4 \pi^{2}} \int_{-\infty}^{\infty}|\Phi(t)|^{2} V(t) d t \\
& =\int_{\infty}^{\infty}\left|\operatorname{li} \cdot{ }_{N \rightarrow \infty} \cdot \int_{-N}^{N} \phi(x) e^{\imath x t} d x\right|^{2} W(t) d t,
\end{aligned}
$$

where

$$
W(t)=\frac{\chi(t ;[a+c, a+d])}{t-(a+c)}+\frac{\chi(t ;[a+d, b+c])}{d-c}+\frac{\chi(t ;[b+c, b+d])}{b+d-t} .
$$

See $[5,6]$. From the property of $(2.7)$, we, in particular, obtain the following inequalities.

THEOREM 2.2. For any $f \in H_{(a, b)}$ and $g \in H_{(c, d)}$, we have the inequality

$$
\begin{aligned}
& \int_{a+c}^{b+d}\left|\operatorname{lid.m} . \int_{-N}^{N} f(x) g(x) e^{\imath x t} d x\right|^{2} W(t) d t \\
& \leqq \frac{1}{2 \pi} \int_{a}^{b}\left|1 . \mathrm{i} . \mathrm{m} \cdot \int_{-N}^{N} f(x) e^{\imath x t} d x\right|^{2} d t \\
& \cdot \frac{1}{2 \pi} \int_{c}^{d} \mid \text { 1.i.m. }\left.\int_{-N}^{N} g(x) e^{\imath x t} d x\right|^{2} d t
\end{aligned}
$$


or, for any $F \in L_{2}(a, b)$ and $G \in L_{2}(c, d)$

$$
\int_{a+c}^{b+d}|(F * G)(t)|^{2} W(t) d t \leqq \int_{a}^{b}|F(t)|^{2} d t \int_{c}^{d}|G(t)|^{2} d t .
$$

As a property of the convolution $F^{*} G$, we have

COROLLARY 2.1. The convolution $F * G$ of $F \in L_{2}(a, b)$ and $G \in L_{2}(c, d)$ is expressible in the form

$$
(F * G)(t)=\Phi(t) V(t)
$$

for a function $\Phi$ satisfying

$$
\int_{-\infty}^{\infty}|\Phi(t)|^{2} V(t) d t<\infty
$$

Conversely, for any $\Phi$ satisfying (2.16), the right hand in (2.15) is expressible in the form, for $F_{j} \in L_{2}(a, b)$ and $G_{j} \in L_{2}(c, d)$

$$
\Phi(t) V(t)=\sum_{j=1}^{\infty}\left(F_{j} * G_{j}\right)(t)
$$

in the sense of the strong convergence in the norm (2.16).

Further, when $G \equiv 1$ on $[0, d]$, we have

COROLlARy 2.2. For any $F \in L_{2}(a, b)$ and for any $d$ such that $a+d \leqq b$, we have the inequality

$$
\begin{aligned}
& \int_{a}^{a+d} \frac{1}{t-a}\left|\int_{a}^{t} F\left(t_{1}\right) d t_{1}\right|^{2} d t+\frac{1}{d} \int_{a+d}^{b}\left|\int_{t-d}^{t} F\left(t_{1}\right) d t_{1}\right|^{2} d t \\
& \quad+\int_{b}^{b+d} \frac{1}{b+d-t}\left|\int_{t-d}^{b} F\left(t_{1}\right) d t_{1}\right|^{2} d t \leqq d \int_{a}^{b}|F(t)|^{2} d t .
\end{aligned}
$$

Further, when $a=c=0$ and $b=d>0$, we have

$$
\int_{0}^{b} \frac{1}{t}\left|\int_{0}^{t} F\left(t_{1}\right) d t_{1}\right|^{2} d t+\int_{b}^{2 b} \frac{1}{2 b-t}\left|\int_{t-b}^{b} F\left(t_{1}\right) d t_{1}\right|^{2} d t \leqq b \int_{0}^{b}|F(t)|^{2} d t .
$$

Corollay 2.2 will give a natural relationship between the magnitudes of the integrals

$$
\left|\int_{a}^{t} F\left(t_{1}\right) d t_{1}\right|^{2} \text { and } \int_{a}^{b}|F(t)|^{2} d t
$$

in a sense. Cf. Hardy-Littlewood-Polya [3, pp. 239-246].

In particular, when $(a, b)=(-a, a)$, we have

$$
K_{(-a, a)}(z, \bar{u})=\frac{\sin (a z-a \bar{u})}{\pi(z-\bar{u})}
$$


and

$$
\int_{-\infty}^{\infty}|f(x)|^{2} d x=\frac{1}{2 \pi} \int_{-a}^{a}|F(t)|^{2} d t
$$

See, for example, de Branges [2, pp. 46-48]. Hence, we have

CoROllary 2.3. For any $f$ and $g \in H_{(-a, a)}$, we have the inequality

$$
\int_{-2 a}^{2 a} \frac{1}{2 a-|t|}\left|\operatorname{li} \operatorname{in}_{N \rightarrow \infty} . \int_{-N}^{N} f(x) g(x) e^{\imath x t} d x\right|^{2} d t \leqq \int_{-\infty}^{\infty}|f(x)|^{2} d x \int_{-\infty}^{\infty}|g(x)|^{2} d x .
$$

Further, for any $F$ and $G \in L_{2}(-\infty, \infty)$ and for any $a>0$, we have the inequalıty

$$
\int_{-2 a}^{2 a} \frac{1}{2 a-|t|}\left|\left(F^{*} G\right)(t)\right|^{2} d t \leqq \int_{-a}^{a}|F(t)|^{2} d t \int_{-a}^{a}|G(t)|^{2} d t
$$

\section{Equality problems.}

We will consider the equality problems for the inequalities obtained in $\S 2$. Note that there does, in general, not exist a general treatment for the equality problem in (2.7). See [4] for some general discussions for this equality problem. But, in the present case we obtain directly

THEOREM 3.1. In the inequality (2.14), equality holds for $F \in L_{2}(a, b)$ and $G \in L_{2}(c, d)$ if and only if $F$ and $G$ are expressible in the form

$$
F(t)=C_{1} e^{i \bar{u} t} \text { on }[a, b] \text { and } G(t)=C_{2} e^{i \bar{u} t} \text { on }[c, d]
$$

for some constants $C_{1}$ and $C_{2}$, and for some point $u \in C$.

Hence, further, equality holds in (2.13) for $f \in H_{(a, b)}$ and $g \in H_{(c, d)}$ if and only if $f$ and $g$ are expressible in the from

$$
f(z)=C_{1} K_{(a, b)}(z, \bar{u}) \quad \text { and } \quad g(z)=C_{2} K_{(c, d)}(z, \bar{u}) .
$$

Proof. We will consider the equality problem in the inequality (2.14). Note that the inequality (2.14) is directly derived as follows:

$$
\begin{aligned}
\int_{a+c}^{b+d}|(F * G)(t)|^{2} W(t) d t & =\int_{a+c}^{a+d} \frac{1}{t-(a+c)}\left|\int_{a}^{t-c} F\left(t_{1}\right) G\left(t-t_{1}\right) d t_{1}\right|^{2} d t \\
& +\int_{a+d}^{b+c} \frac{1}{d-c}\left|\int_{t-d}^{t-c} F\left(t_{1}\right) G\left(t-t_{1}\right) d t_{1}\right|^{2} d t \\
& +\int_{b+c}^{b+d} \frac{1}{b+d-t}\left|\int_{t-d}^{b} F\left(t_{1}\right) G\left(t-t_{1}\right) d t_{1}\right|^{2} d t \\
\leqq & \int_{a+c}^{a+d}\left(\int_{a}^{t-c}\left|F\left(t_{1}\right) G\left(t-t_{1}\right)\right|^{2} d t_{1}\right) d t
\end{aligned}
$$




$$
\begin{aligned}
& +\int_{a+d}^{b+c}\left(\int_{t-d}^{t-c}\left|F\left(t_{1}\right) G\left(t-t_{1}\right)\right|^{2} d t_{1}\right) d t \\
& +\int_{b+c}^{b+d}\left(\int_{t-d}^{b}\left|F\left(t_{1}\right) G\left(t-t_{1}\right)\right|^{2} d t_{1}\right) d t \\
& =\int_{a}^{b}|F(t)|^{2} d t \int_{c}^{d}|G(t)|^{2} d t .
\end{aligned}
$$

Hence, equality holds here if and only if

or

$$
F\left(t_{1}\right) G\left(t-t_{1}\right)=H(t)
$$

$$
F\left(t_{1}\right) G\left(t_{2}\right)=H\left(t_{1}+t_{2}\right) \quad \text { on }[a, b] \times[c, d]
$$

for some function $H$ on $[a+c, b+d]$. Hence, from this functional equation, we have the desired result (3.1).

\section{The case of $L_{2}(-\infty, \infty)$.}

Next, we will consider the case of $F, G \in L_{2}(-\infty, \infty)$. Then, for any $a>0$ and for the restriction of $F$ and $G$ to $[-a, a]$ we can consider the functions

$$
f_{a}(z)=\frac{1}{2 \pi} \int_{-a}^{a} F(t) e^{-\imath z t} d t \text { and } g_{a}(z)=\frac{1}{2 \pi} \int_{-a}^{a} G(t) e^{-\imath z t} d t .
$$

Then, we note that the norms

$$
\left\|f_{a} g_{a}\right\|_{[H(-a, a) \otimes H(-a, a)]_{R}}
$$

do not decrease for $a>0$ and so the limit

$$
\begin{aligned}
\lim _{a \rightarrow \infty} \int_{-2 a}^{2 a} \frac{1}{2 a-|t|}\left|\left(F^{*} G\right)(t)\right|^{2} d t & =\lim _{a \rightarrow \infty}\left\{\int_{-2 a}^{0} \frac{1}{t+2 a}\left|\int_{-a}^{t+a} F\left(t_{1}\right) G\left(t-t_{1}\right) d t_{1}\right|^{2} d t\right. \\
& \left.+\int_{0}^{2 a} \frac{1}{2 a-t}\left|\int_{t-a}^{a} F\left(t_{1}\right) G\left(t-t_{1}\right) d t_{1}\right|^{2} d t\right\}
\end{aligned}
$$

exists.

In order to show this fact, we consider the expression, for any $0<a<b$

$$
\begin{aligned}
f_{b}(z) & =\frac{1}{2 \pi} \int_{-a}^{a} F(t) e^{-\imath z t} d t+\frac{1}{2 \pi} \int_{-b}^{-a} F(t) e^{-\imath z t} d t+\frac{1}{2 \pi} \int_{a}^{b} F(t) e^{-\imath z t} d t \\
: & =f_{a}(z)+f_{(-b,-a)}(z)+f_{(a, b)}(z)
\end{aligned}
$$

and the corresponding reproducing kernels

$$
K_{(-b, b)}(z, \bar{u})=K_{(-a, a)}(z, \bar{u})+K_{(-b,-a)}(z, \bar{u})+K_{(a, b)}(z, \bar{u}) .
$$

These mean that 


$$
H_{(-b, b)}=H_{(-a, a)} \oplus H_{(-b,-a)} \oplus H_{(a, b)}
$$

and

$$
\left\|f_{b}\right\|_{H(-b, b)}^{2}=\left\|f_{a}\right\|_{H(-a, a)}^{2}+\left\|f_{(-b,-a)}\right\|_{H(-b,-a)}^{2}+\left\|f_{(a, b)}\right\|_{H(a, b)}^{2} .
$$

Note that in this case the sum is a direct sum. See [1, pp. 352-354]. From (4.2), we have the identity

$$
\begin{aligned}
K_{(-b, b)}(z, \bar{u})^{2} & =\left(K_{(-a, a)}(z, \bar{u})+K_{(-b,-a)}(z, \bar{u})+K_{(a, b)}(z, \bar{u})\right)^{2} \\
& =K_{(-a, a)}(z, \bar{u}) K_{(-a, a)}(z, \bar{u})+\cdots+K_{(a, b)}(z, \bar{u}) K_{(a, b)}(z, \bar{u})
\end{aligned}
$$

and the corresponding expression

$$
\begin{aligned}
f_{b}(z) g_{b}(z) & =\left(f_{a}(z)+f_{(-b,-a)}(z)+f_{(a, b)}(z)\right)\left(g_{a}(z)+g_{(-b,-a)}(z)+g_{(a, b)}(z)\right) \\
& =f_{a}(z) g_{a}(z)+\cdots+f_{(a, b)}(z) g_{(a, b)}(z) .
\end{aligned}
$$

From these identities we obtain conversely the corresponding identities to (4.3) and (4.4).

$$
\left[H_{(-b, b)} \otimes H_{(-b, b)}\right]_{R}=\left[H_{(-a, a)} \otimes H_{(-a, a)}\right]_{R} \oplus \cdots \oplus\left[H_{(a, b)} \otimes H_{(a, b)}\right]_{R} .
$$

and

$$
\begin{aligned}
& \left\|f_{b} g_{b}\right\|_{[H(-b, b) \otimes H(-b, b)]_{R}}^{2} \\
& \quad=\left\|f_{a} g_{a}\right\|_{[H(-a, a)}^{2} \otimes H_{(-a, a)]_{R}}+\cdots+\left\|f_{(a, b)} g_{(a, b)}\right\|_{\left[H(a, b) \otimes H_{(a, b)}\right]_{R}}^{2} .
\end{aligned}
$$

Hence, in particular, we obtain the desired result

$$
\left\|f_{a} g_{a}\right\|_{[H(-a, a) \otimes H(-a, a)]_{R}} \leqq\left\|f_{b} g_{b}\right\|_{\left[H(-b, b) \otimes H_{(-b, b)]_{R}}\right.} .
$$

Hence, in the inequality (2.20), we obtain the fundamental

Theorem 4.1. For any $F$ and $G \in L_{2}(-\infty, \infty)$, we have the inequality

$$
\lim _{a \rightarrow \infty} \int_{-2 a}^{2 a} \frac{1}{2 a-|t|}\left|\left(F^{*} G\right)(t)\right|^{2} d t \leqq \int_{-\infty}^{\infty}|F(t)|^{2} d t \int_{-\infty}^{\infty}|G(t)|^{2} d t .
$$

Equality does not hold here for $F, G \neq 0$ as functions of $L_{2}(\infty, \infty)$.

The equality statement in this theorem follows from the proof of Theorem 3.1. Of course, we can obtain the corresponding results for iterated convolutions by a similar method, but the results are more complicated than the case of $L_{2}(0, \infty)$. See [7].

\section{REFERENCES}

[1] N. Aronszajn, The theory of reproducing kernels, Trans. Amer. Math. Soc., 68 (1950), 337-404. 
[2] L. De Branges, Hilbert spaces of entire functions, Prentice-Hall, Englewood Cliffs. New Jersey 1968.

[3] G. H. Hardy, J. E. Littlewood And G. Polya, Inequalities, Cambridge University Press. 1967.

[4] S. SAITOH, Reproducing kernels of the direct product of two Hilbert spaces, Riazi J. Kar. Math. Assoc., 4 (1982), 1-20.

[5] S. SAITOH, Integral transforms in Hilbert spaces, Proc. Japan Acad., 58 (1982), 361-364.

[6] S. Saitoh, Hilbert spaces induced by Hilbert space valued functions, Proc. Amer. Math. Soc., 89 (1983), 74-78.

[7] S. SAItoH, A fundamental inequality in the convolution of $L_{2}$ functions on the half line, Proc. Amer. Math. Soc., 91 (1984), 285-286.

[8] E. M. Stein AND G. Weiss, Introduction to Fourier analysis on Euclidean spaces, Princeton University Press. Princeton, N. J. 1971.

Department of Mathematics

FACULTY OF ENGINEERING

GunMa UNIVERSITY

KIRYU 376, JAPAN 